\title{
Increasing dietary linoleic acid does not increase tissue arachidonic acid content in adults consuming Western-type diets: a systematic review
}

Brian S Rett and Jay Whelan*

\begin{abstract}
Background: Linoleic acid, with a DRI of $12-17 \mathrm{~g} / \mathrm{d}$, is the most highly consumed polyunsaturated fatty acid in the Western diet and is found in virtually all commonly consumed foods. The concern with dietary linoleic acid, being the metabolic precursor of arachidonic acid, is its consumption may enrich tissues with arachidonic acid and contribute to chronic and overproduction of bioactive eicosanoids. However, no systematic review of human trials regarding linoleic acid consumption and subsequent changes in tissue levels of arachidonic acid has been undertaken.
\end{abstract}

Objective: In this study, we reviewed the human literature that reported changes in dietary linoleic acid and its subsequent impact on changing tissue arachidonic acid in erythrocytes and plasma/serum phospholipids.

Design: We identified, reviewed, and evaluated all peer-reviewed published literature presenting data outlining changes in dietary linoleic acid in adult human clinical trials that reported changes in phospholipid fatty acid composition (specifically arachidonic acid) in plasma/serum and erythrocytes within the parameters of our inclusion/exclusion criteria.

Results: Decreasing dietary linoleic acid by up to $90 \%$ was not significantly correlated with changes in arachidonic acid levels in the phospholipid pool of plasma/serum $(p=0.39)$. Similarly, when dietary linoleic acid levels were increased up to six fold, no significant correlations with arachidonic acid levels were observed $(p=0.72)$. However, there was a positive relationship between dietary gamma-linolenic acid and dietary arachidonic acid on changes in arachidonic levels in plasma/serum phospholipids.

Conclusions: Our results do not support the concept that modifying current intakes of dietary linoleic acid has an effect on changing levels of arachidonic acid in plasma/serum or erythrocytes in adults consuming Western-type diets.

\section{Background}

Arachidonic acid (AA, 20:4n-6) is a potent bioactive molecule. When released from membrane phospholipids, it is converted to a variety of bioactive compounds, called eicosanoids. These oxidized lipid molecules are related to a number of chronic diseases including cardiovascular disease, cancer and inflammation [1-4]. Enrichment of AA in tissues is positively correlated with

\footnotetext{
* Correspondence: jwhelan@utk.edu

* Correspondence: jwhelan@utk.edu
Department of Nutrition, University of Tennessee, Knoxville, Tennessee 37996-1900, USA
}

$\mathrm{n}-6)$ is the major dietary polyunsaturated fatty acid (PUFA) in the Western diet and is a metabolic precursor to AA, linked biochemically via two desaturases and an elongase. Typical intakes of LA are 12-17 grams per day for women and men, respectively [5], or approximately $6 \%$ of energy. In the absence of other omega- 6 (n-6) PUFA (including dietary AA), dietary LA is the sole contributor to tissue AA. This relationship had been established in experimental rodent models where dietary LA was correlated with tissue AA content in a 
non-linear relationship in rats provided fat-free background diets [6] and lipid-rich diets [7].

Recent reviews suggest this relationship may exist in adult humans consuming a typical Western-type diet $[8,9]$ and some have recommended limiting LA intake as a way to help reduce tissue AA levels $[10,11]$. Certainly, this relationship had been reported in subjects consuming diets containing LA at levels less than $2 \%$ of energy [12]. There are, however, a number of recent papers suggesting that increasing dietary LA does not increase tissue AA levels, but in fact may have an inverse relationship [13,14]. To compound the complexity of this relationship, the family of n-6 PUFA are, in general, synonymously identified to dietary LA, while seemingly ignoring other members who can contribute to tissue AA, i.e., dietary gamma-linolenic acid (GLA, 18:3 n-6) and AA.

This study was designed to explore the relationship of dietary LA and tissue AA, viz., phospholipid pools of plasma/serum and erythrocytes. To our knowledge, this is the first study to review the literature as to whether increasing dietary LA is positively correlated with increasing tissue AA content, and whether reducing dietary LA has the opposite effect in adults consuming Western-type diets. We further investigated what potential contributions other dietary n-6 PUFA may have on tissue AA content. This study was limited in scope and did not address other controversial issues related to dietary LA or other PUFA or their effects on issues related to health.

\section{Methods}

The aim of this paper was to identify, review, and evaluate all peer-reviewed published literature presenting data outlining changes in dietary LA in adult human clinical trials which report phospholipid fatty acid composition (specifically AA) in plasma/serum and erythrocytes. We chose the phospholipid pool in plasma/serum because here is where a majority of the human data is, it represents membranes of lipoproteins derived from the surface of hepatic endoplasmic reticulum [15] (it helps to control for potential variations in other components, such as circulating triglycerides). The studies reporting the fatty acid composition of erythrocyte phospholipids do so because fatty acids in erythrocytes are almost totally esterified in phospholipids. Further refinements to the search strategy included reported changes in tissue AA levels following dietary intake of AA and its various n-6 PUFA precursors, i.e., LA and GLA. Published articles meeting eligibility criteria from 1970 to present were reviewed, of which 4336 articles were retrieved from May 2009 - November 2009 (Figure 1). The primary search engine used was PubMed.gov (The National Library of Medicine, National Institutes of
Health), along with several prominent nutrition-based clinical journals, i.e., American Journal of Clinical Nutrition, British Journal of Nutrition, and any additional citations in articles reviewed. The search terms included linoleic acid, $\gamma$-linolenic acid, gamma-linolenic acid, arachidonic acid, omega- $6, n-6$, olive oil, soybean oil, sunflower oil, safflower oil, corn oil, omega-3, n-3, plasma, erythrocyte, red blood cell and phospholipid.

The following eligibility criteria applied to all accepted articles. Subjects had to be 18 years or older with no known metabolic disorder that would influence tissue AA content. Sufficient data on LA, GLA and/or AA consumption (pre- and post-intervention) was required. The nature of the intervention (i.e., capsules, oils or dietary modifications) had to be presented. The fatty acid data (plasma/serum and/or erythrocyte) had to be determined from fasting patients, pre- and post-supplementation. Baseline and post-treatment of tissue phospholipid fatty acid composition had to be provided. On occasion, percent changes in tissue fatty acid composition were provided and this data was used. Only those papers published after 1970 due to improved gas chromatographic methods were accepted. Articles were automatically excluded if subjects were less than 18 years old, pregnant or nursing, consuming supplements containing long chain $\mathrm{n}-3$ fatty acids or supplemented fish intake above and beyond their typical dietary regimen, or using known inhibitors of AA metabolism, such as non-steroidal anti-inflammatory drugs (NSAIDs).

After an initial review of the papers, 4043 were excluded because of insufficient data or studies that did not investigate our parameters. Of the 293 papers that passed the initial review process, each was reviewed by two independent investigators (BR and JW) and thirty-six were acceptable by both reviewers. Those papers that were not accepted $(\mathrm{n}=249)$ were rejected because baseline data was not sufficiently reported, data for target tissues was not presented, insufficient data was present and did not allow for appropriate calculations, background diets were not sufficiently described, or they included supplementation of restricted food items (i.e., long chain n-3 PUFA). Thirty-six articles were found to meet all of the inclusion-exclusion criteria.

Once accepted, data on dietary n-6 fatty acid intake (\% of calories or $\mathrm{g} / \mathrm{d}$ ) and tissue AA content were extracted. Study design, number and gender of subjects, method of supplementation (i.e., type of oil, capsules or food component used) were recorded. Those studies involving dietary LA as percentage of calories or $\mathrm{g} / \mathrm{d}$ and its effects on changes in tissue AA content are summarized in Tables 1, 2, \&3. Similarly, those studies involving dietary GLA (0.36-6.00 g/d) and AA (0.50-6.00 g/d) are summarized in Tables 4 and 5 . If a study met the 


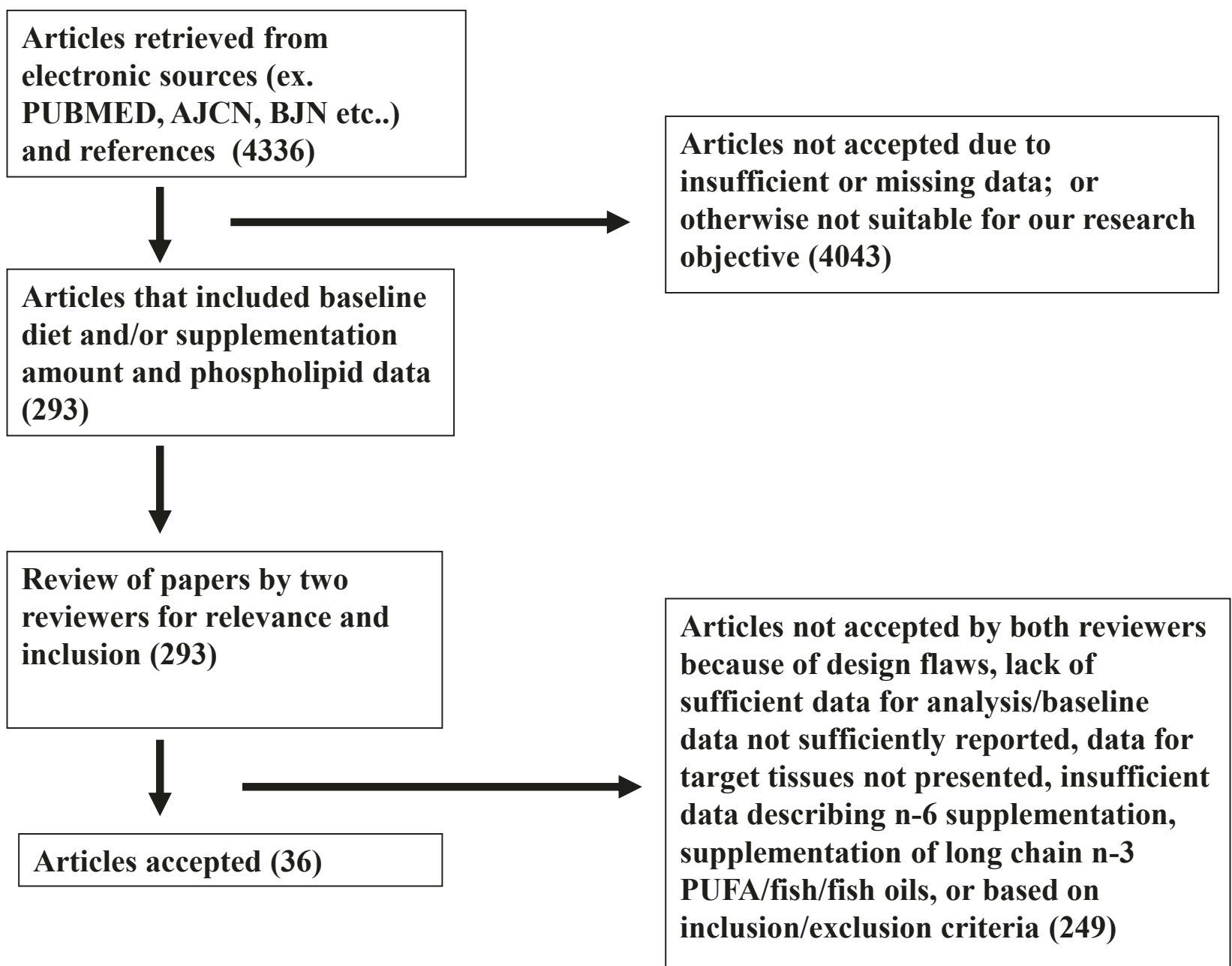

Figure 1 Schematic outlining the systematic review.

eligibility criteria and contained more than one comparison, each comparison was reported as a separate data point.

Baseline tissue AA levels were defined as relative abundance of AA in tissue phospholipids prior to dietary supplementation (or reduction) of the corresponding dietary n-6 PUFA of interest (i.e., LA, GLA or AA). In the case of cross-over designs, baseline fatty acid composition was established following a washout period or after supplementation of a control diet if there were no or only minor changes in the dietary n-6 PUFA content. For example, a supplement rich in oleic acid (a monounsaturated fatty acid typically used as a control and known to have a neutral effect on tissue AA content) could be used as a control lipid (or oil) prior to supplementation of an equal amount of a lipid (or oil) rich in LA. Percent change for each dietary n-6 fatty acid of interest was used to standardize the relative differences between baseline intakes and intervention intakes following the experimental period using the following formula:

$$
\frac{\text { intervention intake - baseline intake }}{\text { baseline intake }} \times 100
$$

The levels of intake were based on the relative caloric amount (\% of calories), and when this data was not available absolute intake levels $(\mathrm{g} / \mathrm{d})$ were used. Percent change for tissue AA content was used to standardize the relative differences between baseline levels and intervention levels following the experimental period using the following formula:

$$
\frac{\text { post }- \text { intervention level - baseline level }}{\text { baseline level }} \times 100
$$


Table 1 Studies outlining the effects of decreasing dietary linoleic acid levels (\% energy) from baseline on changes in plasma/serum phospholipid arachidonic acid level

\begin{tabular}{|c|c|c|c|c|c|c|c|}
\hline $\begin{array}{l}\text { Author, } \\
\text { (reference) }\end{array}$ & Study design & Subjects & $\begin{array}{l}\text { Diet } \\
\text { length }\end{array}$ & Diet comparison & $\begin{array}{l}\text { LA (\% change) } \\
\text { Baseline to } \\
\text { intervention }\end{array}$ & $\begin{array}{l}\triangle A A(\%) \\
\text { change }\end{array}$ & Comments \\
\hline \multirow[t]{2}{*}{ Lasserre [17] } & $\begin{array}{l}\text { Randomized } \\
\text { crossover }\end{array}$ & $N=24$ & 5 months & $\begin{array}{l}\text { Peanut oil diet (baseline) } \\
\text { compared to milk fat diet }\end{array}$ & $-90(6.5 \%-0.6 \%)$ & 9.99 N.S. & $\begin{array}{l}\text { Used peanut oil group b/c close to DRI for LA. Subjects were nuns in } \\
\text { monastery. }\end{array}$ \\
\hline & & & & $\begin{array}{l}\text { Peanut oil diet (baseline) } \\
\text { compared to low erucic acid } \\
\text { rapeseed oil (canola oil) diet }\end{array}$ & $-30(6.5 \%-4.5 \%)$ & -5.49 N.S. & $\begin{array}{l}\text { Used peanut oil group b/c close to DRI for LA. Subjects were nuns in } \\
\text { monastery. }\end{array}$ \\
\hline $\begin{array}{l}\text { Lichtenstein } \\
\text { [35] }\end{array}$ & $\begin{array}{l}\text { Randomized } \\
\text { double-blind } \\
\text { crossover }\end{array}$ & $N=30$ & 35 days & $\begin{array}{l}\text { Soybean oil diet (baseline) } \\
\text { compared to high oleic acid } \\
\text { soybean oil diet }\end{array}$ & $-82(11 \%-1.9 \%)$ & -2.58 N.S. & $\begin{array}{l}\text { Pooled data of men and women. Baseline diet of } 10.96 \% \text { energy } \\
\text { closest to DRI for LA. AA did not differ among remaining groups } \\
\text { tested either }\end{array}$ \\
\hline Liou [37] & $\begin{array}{l}\text { Randomized } \\
\text { crossover }\end{array}$ & $N=24$ & 4 weeks & $\begin{array}{l}\text { High linoleic acid sunflower oil } \\
\text { (diet) compared to high oleic } \\
\text { acid safflower oil (diet) }\end{array}$ & $-63(10.5 \%-3.8 \%)$ & -5.55 N.S. & $\begin{array}{l}\text { Incorporated test oils into baked foods (cookies, breads), mayonnaise, } \\
\text { salad dressing. AA data presented in graphs, not tables. Fish intake } \\
\text { was avoided for all groups. AA PL content did not differ between } \\
\text { sequence of diets going from high LA to low LA or vice versa. Study } \\
\text { address low or high LA with constant ALA at } 1 \% \text {. }\end{array}$ \\
\hline Goyens [38] & $\begin{array}{l}\text { Double-blind } \\
\text { intervention }\end{array}$ & $N=19$ & 6 weeks & $\begin{array}{l}\text { Reduced LA in food items } \\
\text { (margarines, pastries, baked } \\
\text { goods) }\end{array}$ & $-57(7 \%-3 \%)$ & 5.26 N.S. & $\begin{array}{l}\text { Test oils consumed in margarine and pastries. Prohibited consumption } \\
\text { of fish or marine foods in all groups. }\end{array}$ \\
\hline $\begin{array}{l}\text { Vega-Lopez } \\
{[36]}\end{array}$ & $\begin{array}{l}\text { Randomized } \\
\text { crossover }\end{array}$ & $N=15$ & 35 days & $\begin{array}{l}\text { Canola oil diet compared to } \\
\text { palm oil diet }\end{array}$ & $-50(6.5 \%-3.3 \%)$ & -8.06 N.S. & $\begin{array}{l}\text { Canola oil in mixed foods was replaced by palm oil in mixed foods. } \\
\text { AA did not change among all three dietary groups. Canola oil diet is } \\
\text { baseline because closest to DRI LA intake. }\end{array}$ \\
\hline \multirow[t]{2}{*}{ Li [39] } & $\begin{array}{l}\text { Parallel } \\
\text { intervention }\end{array}$ & $N=17$ & 28 days & $\begin{array}{l}\text { High LA diet to moderate LA } \\
\text { diet using canola oil/canola } \\
\text { margarine }\end{array}$ & $-48(13.5 \%-7 \%)$ & -3.80 N.S & $\begin{array}{l}\text { Subjects were given diet more than twice DRI for } L A \text { and then given } \\
\text { diet resembling the DRI for LA. All groups were asked to not consume } \\
\text { fish. }\end{array}$ \\
\hline & & & & $\begin{array}{l}\text { High LA diet to normal LA diet } \\
\text { using canola oil/canola } \\
\text { margarine }\end{array}$ & $-39(11.9 \%-7.3 \%)$ & -16.5 N.S. & \\
\hline $\begin{array}{l}\text { Mantzioris } \\
{[40]}\end{array}$ & $\begin{array}{l}\text { Parallel } \\
\text { intervention }\end{array}$ & $N=15$ & 4 weeks & $\begin{array}{l}\text { Control diet (sunflower oil) } \\
\text { group compared to } \\
\text { intervention diet (flaxseed oil) }\end{array}$ & $-57(7.8 \%-3.3 \%)$ & -4.5 N.S. & $\begin{array}{l}\text { Control group consumed relatively close to DRI for LA while } \\
\text { intervention group reduced LA by more than half. }\end{array}$ \\
\hline King [16] & $\begin{array}{l}\text { Randomized } \\
\text { parallel }\end{array}$ & $N=66$ & 6 weeks & $\begin{array}{l}\text { Baseline diet compared to low } \\
\text { fat diet }\end{array}$ & $-29(10 \%-7.1 \%)$ & $4.1(p<0.05)$ & $\begin{array}{l}\text { Used modified food items for diets containing different amounts of } \\
\text { fat. Reported AA PL in \% change. }\end{array}$ \\
\hline Geppert [41] & $\begin{array}{l}\text { Randomized } \\
\text { double- blind } \\
\text { intervention }\end{array}$ & $N=54$ & 8 weeks & $\begin{array}{l}\text { Baseline diet compared to LA } \\
\text { reduced diet (using olive oil } \\
\text { capsules) }\end{array}$ & $-12(5.8 \%-5.1 \%)$ & 1.12 N.S. & Used olive oil capsules with vegetarians. \\
\hline
\end{tabular}

Percent change $( \pm)$ from baseline in AA that is significant is identified with $(p<0.05)$. Percent change $( \pm)$ from baseline in AA that is not significant is denotedby N.S.

Abbreviations: AA, arachidonic acid; DRI, Dietary Reference Intake; LA, linoleic acid; PL, phospholipid 
Table 2 Studies outlining the effects of increasing dietary linoleic acid levels (\% energy) from baseline on changes in plasma/serum phospholipid arachidonic acid level

\begin{tabular}{|c|c|c|c|c|c|c|c|}
\hline $\begin{array}{l}\text { Author, } \\
\text { (reference) }\end{array}$ & $\begin{array}{l}\text { Study } \\
\text { design }\end{array}$ & Subjects & Diet length & Diet comparison & $\begin{array}{c}\text { LA (\% } \\
\text { change) } \\
\text { Baseline to } \\
\text { intervention }\end{array}$ & $\begin{array}{l}\triangle \text { AA (\%) } \\
\text { change }\end{array}$ & Comments \\
\hline King [16] & $\begin{array}{l}\text { Randomized } \\
\text { parallel } \\
\text { intervention }\end{array}$ & $N=33$ & 6 weeks & $\begin{array}{l}\text { Baseline diet compared to moderate fat } \\
\text { diet }\end{array}$ & $\begin{array}{l}13(10.3 \%- \\
1.6 \%)\end{array}$ & $-3.2(p<0.05)$ & $\begin{array}{l}\text { Used modified food items for diets containing different } \\
\text { amounts of fat. Reported AA PL as \% change. }\end{array}$ \\
\hline \multirow[t]{2}{*}{ Thijssen [42] } & $\begin{array}{l}\text { Randomized } \\
\text { multiple } \\
\text { crossover }\end{array}$ & $N=45$ & 5 weeks & $\begin{array}{c}\text { Stearic acid diet to oleic acid diet in } \\
\text { food items (using margarines, breads, } \\
\text { sponge cakes) }\end{array}$ & $\begin{array}{l}14(2.1 \% \\
-2.4 \%)\end{array}$ & -2.24 (N.S.) & $\begin{array}{c}\text { No order to diets given. Subjects received all } 3 \text { diets with } \\
\text { varying amounts of LA. }\end{array}$ \\
\hline & & & & $\begin{array}{l}\text { Oleic Acid to LA food items (margarines, } \\
\text { breads, sponge cakes) }\end{array}$ & $\begin{array}{c}287(2.4 \%- \\
9.3 \%)\end{array}$ & -1.15 (N.S.) & $\begin{array}{c}\text { No order to diets given. Subjects received all } 3 \text { diets with } \\
\text { varying amounts of LA. }\end{array}$ \\
\hline \multirow[t]{2}{*}{$\begin{array}{c}\text { Montoya } \\
{[43]}\end{array}$} & $\begin{array}{l}\text { Sequential } \\
\text { interventions }\end{array}$ & $N=41$ & 4 weeks & $\begin{array}{l}\text { From palm oil based diet compared to } \\
\text { olive oil based diet }\end{array}$ & $16(3.2 \%-3.7 \%)$ & 4.82 (N.S.) & $\begin{array}{l}\text { Used nuns and priests. Everyone consumed same sequence of } \\
\text { diets. AA did not change among the three test diets. No } \\
\text { crossover, subjects were their own controls. }\end{array}$ \\
\hline & & & & $\begin{array}{l}\text { Olive oil based diet to sunflower oil base } \\
\text { diet }\end{array}$ & $\begin{array}{l}230(3.7 \%- \\
12.2 \%)\end{array}$ & -3.44 (N.S.) & \\
\hline \multirow[t]{2}{*}{$\begin{array}{l}\text { Lichtenstein } \\
\quad[35]\end{array}$} & $\begin{array}{l}\text { Randomized } \\
\text { double-blind } \\
\text { crossover }\end{array}$ & $N=30$ & 35 days & $\begin{array}{l}\text { Baseline soybean oil diet compared to } \\
\text { low saturated fat-soybean oil diet. The } \\
\text { soybean oils varied in LA composition }\end{array}$ & $\begin{array}{l}15.8(11 \% \\
-12.7 \%)\end{array}$ & 0.89 (N.S.) & $\begin{array}{l}\text { Pooled data of men and women. Five diets of random order. } \\
\text { Subjects visited metabolic kitchen } 3 \text { times week. Varying LA } \\
\text { amounts using modified soybean oils. }\end{array}$ \\
\hline & & & & $\begin{array}{l}\text { Baseline high oleic-soybean oil diet to } \\
\text { low ALA-soybean oil diet }\end{array}$ & $\begin{array}{l}552(1.9 \%- \\
2.5 \%)\end{array}$ & 1.27 (N.S.) & \\
\hline \multirow[t]{2}{*}{ Li [39] } & $\begin{array}{c}\text { Parallel } \\
\text { intervention }\end{array}$ & $\begin{array}{l}N=10 \\
N=7\end{array}$ & $\begin{array}{l}14 \text { days } 14 \\
\text { days }\end{array}$ & $\begin{array}{l}\text { Baseline Western diet to intervention diet } \\
\text { increased in LA intake using safflower oil }\end{array}$ & $\begin{array}{l}17.8(10.1 \%- \\
11.9 \%)\end{array}$ & 6.18 (N.S.) & $\begin{array}{l}\text { Used safflower oil/safflower margarine to increase LA in diet to } \\
\text { almost twice DRI of LA. AA did not differ among all groups in } \\
\text { study. }\end{array}$ \\
\hline & & & & $\begin{array}{l}\text { Baseline Western diet to intervention diet } \\
\text { increased in LA intake using safflower oil }\end{array}$ & $\begin{array}{l}82.4(7.4 \%- \\
13.5 \%)\end{array}$ & 0.96 (N.S.) & $\begin{array}{l}\text { Used safflower oil/safflower margarine to increase LA in diet to } \\
\text { more than twice DRI of LA. AA did not differ among all groups } \\
\text { in study. }\end{array}$ \\
\hline $\begin{array}{l}\text { Vega-Lopez } \\
{[36]}\end{array}$ & $\begin{array}{l}\text { Randomized } \\
\text { crossover }\end{array}$ & $N=15$ & 35 days & $\begin{array}{l}\text { Canola oil diet compared to soybean oil } \\
\text { diet }\end{array}$ & $\begin{array}{l}64.2(6.5 \%- \\
10.7 \%)\end{array}$ & -2.35 (N.S.) & $\begin{array}{l}\text { Canola oil in mixed foods was replaced by soybean oil in mixed } \\
\text { foods. AA did not change among all three dietary groups. } \\
\text { Canola oil diet is baseline because close to the DRI for LA. }\end{array}$ \\
\hline Liou [37] & $\begin{array}{l}\text { Randomized } \\
\text { crossover }\end{array}$ & $N=22$ & 4 weeks & $\begin{array}{l}\text { Low LA diet (high in oleic acid safflower } \\
\text { oil) compared to high LA diet (high in } \\
\text { sunflower oil) }\end{array}$ & $\begin{array}{c}176(3.8 \%- \\
10.5 \%)\end{array}$ & -0.18 (N.S.) & $\begin{array}{c}\text { AA PL content presented as graphs, not numerically. Fish was } \\
\text { avoided in all dietary groups. ALA intake was kept constant } \\
\text { between low/high diet. }\end{array}$ \\
\hline \multirow[t]{2}{*}{ Valsta [19] } & $\begin{array}{l}\text { Randomized } \\
\text { crossover }\end{array}$ & $N=39$ & 6 weeks & $\begin{array}{l}\text { Habitual diet compared to high LA } \\
\text { trisunflower oil diet }\end{array}$ & $86(4.2 \%-7.8 \%)$ & $10(p<0.05)$ & $\begin{array}{l}\text { Fish cut in half in all dietary groups. Has a baseline for before } \\
\text { each diet. Used trisunflower oil in margarine, food oil, salad } \\
\text { dressing, bread, cake and cookies, in place of habitual foods. }\end{array}$ \\
\hline & & & & $\begin{array}{c}\text { Habitual diet compared to high ALA } \\
\text { rapeseed oil diet }\end{array}$ & $45(4.2 \%-6.1 \%)$ & 2.77 (N.S.) & \\
\hline Raatz [18] & $\begin{array}{l}\text { Randomized } \\
\text { crossover }\end{array}$ & $N=10$ & 28 days & $\begin{array}{c}\text { Low fat diet ( } 20 \% \text { energy) compared to } \\
\text { high fat diet ( } 45 \% \text { energy). }\end{array}$ & 100 (6\%-12\%) & $-16(p<0.05)$ & $\begin{array}{c}\text { Modified foods rich in LA. Random order to diet, so baseline } \\
\text { was chosen based on DRI of LA. Used washout period of 21-28 } \\
\text { days }\end{array}$ \\
\hline Lasserre [17] & $\begin{array}{l}\text { Randomized } \\
\text { crossover }\end{array}$ & $N=24$ & 5 months & $\begin{array}{c}\text { Peanut oil diet compared to sunflower } \\
\text { oil }\end{array}$ & $\begin{array}{l}111(6.5 \%- \\
13.7 \%)\end{array}$ & $-20(p<0.05)$ & Used peanut oil group b/c close to DRI for LA. \\
\hline Innis [13] & $\begin{array}{l}\text { Randomized } \\
\text { crossover }\end{array}$ & $N=24$ & 8 weeks & Low LA diet to high LA diet & $\begin{array}{c}176(3.8 \%- \\
10.5 \%)\end{array}$ & 1.86 (N.S.) & Controlled for dietary AA. \\
\hline
\end{tabular}


Table 3 Studies outlining the effects of supplementing dietary linoleic acid levels (g/day) on changes in plasma/serum phospholipid arachidonic acid level

\begin{tabular}{|c|c|c|c|c|c|c|}
\hline $\begin{array}{c}\text { Author, } \\
\text { (reference) }\end{array}$ & Study design & Subjects & $\begin{array}{l}\text { Diet } \\
\text { length }\end{array}$ & $\begin{array}{l}\text { LA source, amount } \\
\text { supplemented }(\mathrm{g} / \mathrm{d})\end{array}$ & $\begin{array}{l}\triangle A A(\%) \\
\text { change }\end{array}$ & Comments \\
\hline \multirow[t]{2}{*}{$\begin{array}{l}\text { Anderson } \\
{[44]}\end{array}$} & Parallel intervention & $N=8$ & $\begin{array}{c}3 \\
\text { months }\end{array}$ & Olive oil (0.2) & 8.46 (N.S.) & Olive oil supplement \\
\hline & & $N=9$ & & Olive oil (0.2) & 9.05 (N.S.) & $\begin{array}{c}\text { Provided LA for two differen } \\
\text { groups }\end{array}$ \\
\hline \multirow[t]{2}{*}{ Thies [45] } & $\begin{array}{l}\text { Randomized, double-blind, } \\
\text { parallel intervention }\end{array}$ & $N=8$ & $\begin{array}{c}12 \\
\text { weeks }\end{array}$ & Oil blend (0.64) & 3.38 (N.S.) & $\begin{array}{l}\text { Different oil blends were } \\
\text { sources of } L A\end{array}$ \\
\hline & & & & Placebo oil (0.9) & 5.25 (N.S.) & \\
\hline Geppert [21] & $\begin{array}{l}\text { Randomized double- blind } \\
\text { parallel intervention }\end{array}$ & $N=20$ & 8 weeks & Oil blend (0.86) & $-7(p<0.05)$ & $\begin{array}{l}\text { Blend palm, rapeseed and } \\
\text { sunflower oil }\end{array}$ \\
\hline $\begin{array}{c}\text { Johansson } \\
{[46]}\end{array}$ & $\begin{array}{c}\text { Randomized, double-blind, } \\
\text { crossover }\end{array}$ & $N=12$ & 4 weeks & $\begin{array}{l}\text { Sea buckthorn berry oil } \\
(0.90)\end{array}$ & -2.2 (N.S.) & $\begin{array}{c}\text { Sea buckthorn berry oil is } \\
17.9 \% \text { LA. }\end{array}$ \\
\hline Kew [47] & $\begin{array}{l}\text { Double-blind, parallel } \\
\text { intervention }\end{array}$ & $N=42$ & 4 weeks & Olive oil (0.92) & -13.04 (N.S.) & \\
\hline Buckley [48] & $\begin{array}{l}\text { Double-blind parallel } \\
\text { intervention }\end{array}$ & $N=45$ & 4 weeks & Olive oil (0.95) & -7.14 (N.S.) & \\
\hline \multirow[t]{3}{*}{ Yaqoob [34] } & $\begin{array}{l}\text { Randomized, double- blind } \\
\text { parallel intervention }\end{array}$ & $\begin{array}{l}N=8 \text { per } \\
\text { group }\end{array}$ & $\begin{array}{c}12 \\
\text { weeks }\end{array}$ & $\begin{array}{l}\text { Placebo (coconut/soybean } \\
\text { oil) (1.0) }\end{array}$ & 20 (N.S.) & \\
\hline & & & & Olive oil (1.2) & -2.1 (N.S.) & \\
\hline & & & & Sunflower oil (6.95) & 16 (N.S.) & \\
\hline \multirow[t]{2}{*}{ Wallace [49] } & $\begin{array}{l}\text { Randomized, double- blind } \\
\text { parallel intervention }\end{array}$ & $N=8$ & $\begin{array}{c}12 \\
\text { weeks }\end{array}$ & Oil blend (1.52) & -6.97 (N.S.) & \\
\hline & & $N=8$ & & palm/soybean oil (1.7) & 2.43 (N.S.) & \\
\hline Miles [27] & $\begin{array}{l}\text { Randomized, double-blind } \\
\text { parallel intervention }\end{array}$ & $N=8$ & $\begin{array}{c}12 \\
\text { weeks }\end{array}$ & $\begin{array}{c}\text { Placebo (palm/sunflower oil) } \\
(2.07)\end{array}$ & 2.19 (N.S.) & \\
\hline $\begin{array}{l}\text { Grimsgaard } \\
{[20]}\end{array}$ & $\begin{array}{l}\text { Double-blind, parallel } \\
\text { intervention }\end{array}$ & $N=78$ & 7weeks & Corn oil (2.24) & $\begin{array}{c}3.1(\mathrm{p}< \\
0.05)\end{array}$ & \\
\hline Conquer [50] & $\begin{array}{l}\text { Double-blind, parallel } \\
\text { intervention }\end{array}$ & $N=24$ & 42 days & Corn oil (2.39) & 1.12 (N.S.) & \\
\hline Finnegan [51] & $\begin{array}{l}\text { Double-blind, parallel } \\
\text { intervention }\end{array}$ & $N=50$ & $\begin{array}{c}6 \\
\text { months }\end{array}$ & Safflower/sunflower (11.6) & 7.19 (N.S.) & $\begin{array}{l}\text { Test oils provided as } \\
\text { margarine and capsules }\end{array}$ \\
\hline
\end{tabular}

Percent change $( \pm)$ from baseline in AA that is significant is identified with $(p<0.05)$. Percent change $( \pm)$ from baseline in AA that is not significant is denoted by N.S. Abbreviations: AA, arachidonic acid; DRI, Dietary Reference Intake; LA, linoleic acid

Table 4 Studies outlining the effects of supplementing dietary gamma-linolenic acid on changes in plasma/serum phospholipid arachidonic acid level

\begin{tabular}{|c|c|c|c|c|c|c|}
\hline $\begin{array}{c}\begin{array}{c}\text { Author, } \\
\text { (reference) }\end{array} \\
\end{array}$ & Study design & Subjects & $\begin{array}{c}\text { Diet } \\
\text { length }\end{array}$ & $\begin{array}{l}\text { GLA source, amount } \\
\text { supplemented }(g / d)\end{array}$ & $\begin{array}{l}\triangle A A(\%) \\
\text { change }\end{array}$ & Comments \\
\hline Ebden [52] & $\begin{array}{l}\text { Double-blind } \\
\text { intervention }\end{array}$ & $N=6$ & $\begin{array}{c}8 \\
\text { weeks }\end{array}$ & Efamol oil (0.36) & 6.1 (N.S.) & $\begin{array}{l}\text { No crossover with placebo. Subjects were asthmatics } \\
\text { used medication or bronchodilator. }\end{array}$ \\
\hline $\begin{array}{l}\text { Thavonen } \\
\text { [29] }\end{array}$ & $\begin{array}{l}\text { Randomized, double- } \\
\text { blind crossover }\end{array}$ & $N=15$ & $\begin{array}{c}3 \\
\text { weeks }\end{array}$ & $\begin{array}{l}\text { Black current seed oil } \\
\qquad(0.38)\end{array}$ & 3.7 (N.S.) & Subjects aged $55-75$ years old \\
\hline Theis [45] & $\begin{array}{l}\text { Double-blind parallel } \\
\text { interventions }\end{array}$ & $N=8$ & $\begin{array}{c}12 \\
\text { weeks }\end{array}$ & $\begin{array}{l}\text { GLA-rich triacylglycerol } \\
\text { capsules }(0.77)\end{array}$ & $\begin{array}{c}27(p< \\
0.05)\end{array}$ & $\begin{array}{l}\text { Subjects consumed capsules for } 12 \text { weeks. AA } \\
\text { changed only on } 12^{\text {th }} \text { week. }\end{array}$ \\
\hline $\begin{array}{l}\text { Yaqoob } \\
{[34]}\end{array}$ & $\begin{array}{l}\text { Double-blind parallel } \\
\text { intervention }\end{array}$ & $N=8$ & $\begin{array}{c}12 \\
\text { weeks }\end{array}$ & $\begin{array}{l}\text { Evening primrose oil } \\
\text { (1.06) }\end{array}$ & 14 (N.S.) & \\
\hline Mills [28] & $\begin{array}{l}\text { Randomized double- } \\
\text { blind parallel } \\
\text { intervention }\end{array}$ & $N=10$ & $\begin{array}{c}28 \\
\text { days }\end{array}$ & Borage oil (1.30) & $\begin{array}{c}12(p< \\
0.05)\end{array}$ & $\begin{array}{l}\text { AA data available for only pre and post intervention } \\
\qquad \text { (28 days) }\end{array}$ \\
\hline Miles [27] & $\begin{array}{l}\text { Randomized double- } \\
\text { blind intervention }\end{array}$ & $N=8-12$ & $\begin{array}{c}12 \\
\text { weeks }\end{array}$ & $\begin{array}{l}\text { Borage oil capsules } \\
\text { (2.00) }\end{array}$ & $\begin{array}{c}15(p< \\
0.05)\end{array}$ & $\begin{array}{l}\text { Consumed capsules for } 12 \text { weeks. AA only increased } \\
\text { after the 8th week, no difference after } 8^{\text {th }} \text { week. }\end{array}$ \\
\hline $\begin{array}{l}\text { Johnson } \\
\text { [53] }\end{array}$ & Pre-post intervention & $N=5$ & $\begin{array}{c}3 \\
\text { weeks }\end{array}$ & $\begin{array}{l}\text { Ultra-GLA capsules } \\
\qquad(6.00)\end{array}$ & $\begin{array}{c}31(p< \\
0.05)\end{array}$ & \\
\hline
\end{tabular}

Percent change ( \pm ) from baseline in AA that is significant is identified with $(p<0.05)$. Percent change $( \pm)$ from baseline in AA that is not significant is denoted by N.S. Abbreviations: AA, arachidonic acid; GLA, gamma-linolenic acid 
Table 5 Studies outlining the effects of supplementing dietary arachidonic acid on changes in plasma/serum phospholipid arachidonic acid level

\begin{tabular}{|c|c|c|c|c|c|c|}
\hline $\begin{array}{l}\text { Author, } \\
\text { (reference) }\end{array}$ & Study design & Subjects & Diet length & $\begin{array}{l}\text { AA source, amount } \\
\text { supplemented }(g / d)\end{array}$ & $\begin{array}{l}\triangle A A(\%) \\
\text { change }\end{array}$ & Comments \\
\hline Sinclair [54] & Parallel intervention & $N=4$ & 7 days & White meat/eggs (0.50) & $\begin{array}{c}52(p< \\
0.05)\end{array}$ & Consumed $\mathrm{AA}$ rich, low fat diet \\
\hline Ishikura [55] & Double-blind crossover & $N=25$ & 1 month & $\begin{array}{l}\text { SUNTGA40S capsules } \\
(0.72)\end{array}$ & $\begin{array}{c}27(p< \\
0.05)\end{array}$ & Derived from Mortierella alpina \\
\hline Theis [45] & $\begin{array}{l}\text { Randomized, double-blind, } \\
\text { parallel intervention }\end{array}$ & $N=48$ & 12 weeks & ARASCO (0.68) & $\begin{array}{c}85(p< \\
0.05)\end{array}$ & Derived from Mortierella alpina \\
\hline $\begin{array}{c}\text { Kusmoto } \\
{[56]}\end{array}$ & Double-blind intervention & $N=12$ & 4 weeks & SUNTGA40S (0.84) & $\begin{array}{c}45(p< \\
0.05)\end{array}$ & Derived from Mortierella alpina \\
\hline Nelson [57] & $\begin{array}{l}\text { Single blind crossover } \\
\text { intervention }\end{array}$ & $N=10$ & 50 days & ARASCO (1.49) & $\begin{array}{c}85(\mathrm{p}< \\
0.05)\end{array}$ & $\begin{array}{l}\text { Had } 65 \text { day washout period. } \\
\text { Derived from Mortierella alpina }\end{array}$ \\
\hline $\begin{array}{l}\text { Seyberth } \\
\text { [58] }\end{array}$ & Single blind intervention & $N=4$ & $\begin{array}{c}\text { 2-3 weeks, } \\
\text { depending upon } \\
\text { subject }\end{array}$ & $\begin{array}{c}\text { Capsules, AA ethyl ester } \\
(6.00)\end{array}$ & $\begin{array}{c}136(p< \\
0.05)\end{array}$ & Averaged from all 4 subjects \\
\hline
\end{tabular}

Percent change $( \pm)$ from baseline in AA that is significant is identified with $(p<0.05)$. Percent change $( \pm)$ from baseline in AA that is not significant is denoted by N.S.

Abbreviations: $\mathrm{AA}$, arachidonic acid

\section{Statistical Analysis}

The overall linear correlation between percent change of dietary n- 6 fatty acids and percent change of tissue AA was computed using the Proc Corr procedure in SAS 9.2 (SAS Institute Inc. SAS Campus Drive, Cary, North Carolina). The correlation matrix and the $\mathrm{T}$ statistic tested for correlation and statistical significance, respectively. For the linear correlations, the equation of the line was computed, and represented by $\mathrm{y}=\mathrm{mx}$ for those that exhibited linearity. Data not resembling a linear relationship (i.e. dietary GLA and AA) utilized a polynomial growth curve from SAS General Linear Model and t-tests for model parameters tested for significance. Pvalues less than or equal to 0.05 were considered significant. The $\mathrm{Y}$ values represent changes of AA (\% from baseline) and the $X$ values represent the changes of the various dietary n-6 PUFA (\% from baseline or g/d supplemented). In addition to the overall correlation test, the statistical significance for each individual data point (for changes in tissue AA), as reported by the authors in their respective manuscripts, was identified in each graph. If the changes from baseline were significantly different the data was represented by triangle. If the changes from baseline were not statistically different, they were represented by a diamond.

\section{Results}

Eleven comparisons reported decreases in LA intakes $(-12 \%$ to $-90 \%)$ and no significant correlations were associated with changes in plasma/serum phospholipid AA content $\left(\mathrm{r}^{2}=0.07, \mathrm{p}=0.44, \mathrm{y}=0.026 \mathrm{x}\right.$ ) (Table 1 and Figure 2). Only one study of the eleven reported a significant change, a $4.1 \%$ increase in AA content, following a $29 \%$ reduction in LA intake [16].
Increases in dietary LA, ranging from 12\%-550\%, was not significantly correlated with changes in plasma/ serum phospholipid AA content $\left(\mathrm{r}^{2}=0.074, \mathrm{p}=0.45\right.$, $\mathrm{y}$ $=-0.0053 \mathrm{x}$ ) (Table 2 and Figure 3$)$. Of the sixteen comparisons, only four studies reported significant changes in AA levels when dietary LA levels were increased; three studies reported 3-20\% reductions following 12\%$110 \%$ increases in LA consumption [16-18] and only one study reported a significant increase in AA content (10\%) following an $86 \%$ increase in LA intake [19]. Subdividing the studies by design (crossover versus noncrossover) had no effect on the results (data not shown). Similarly, in those studies that only reported absolute levels of LA supplementation (g/d), increasing LA supplementation was not significantly correlated with changes in plasma/serum phospholipid AA content $\left(\mathrm{r}^{2}\right.$ $=0.092, \mathrm{p}=0.64, \mathrm{y}=0.969 \mathrm{x}$ ) (Table 3 and Figure 4 ). Of the seventeen comparisons, only two were significantly different, one resulted in an increase in AA content by $3 \%$ following supplementation of $2.24 \mathrm{~g} / \mathrm{d}$ of LA [20] and the other resulted in a reduction of AA content by $7 \%$ following supplementation of $0.86 \mathrm{~g} / \mathrm{d}$ [21].

Similar comparisons were made in erythrocytes with increasing and decreasing intakes of LA, although the number of studies were more limited. Increases in dietary LA, ranging from $12 \%-100 \%$, were not significantly correlated with changes in tissue AA content $\left(\mathrm{r}^{2}=0.06\right.$, $\mathrm{p}=0.75, \mathrm{y}=-0.1479 \mathrm{x}$ ) (Figure 5). Reducing dietary LA intake $(-12 \%$ to $-70 \%)$ was not significantly correlated with changes in tissue AA content $\left(\mathrm{r}^{2}=0.017, \mathrm{p}=0.77\right.$, $\mathrm{y}=-0.0174 \mathrm{x}$ ) (Figure 6). In addition, out of the seven studies, only one study reported a significant change where decreasing dietary LA intake by $29 \%$ resulted in a $4 \%$ increase in AA content [16]. 


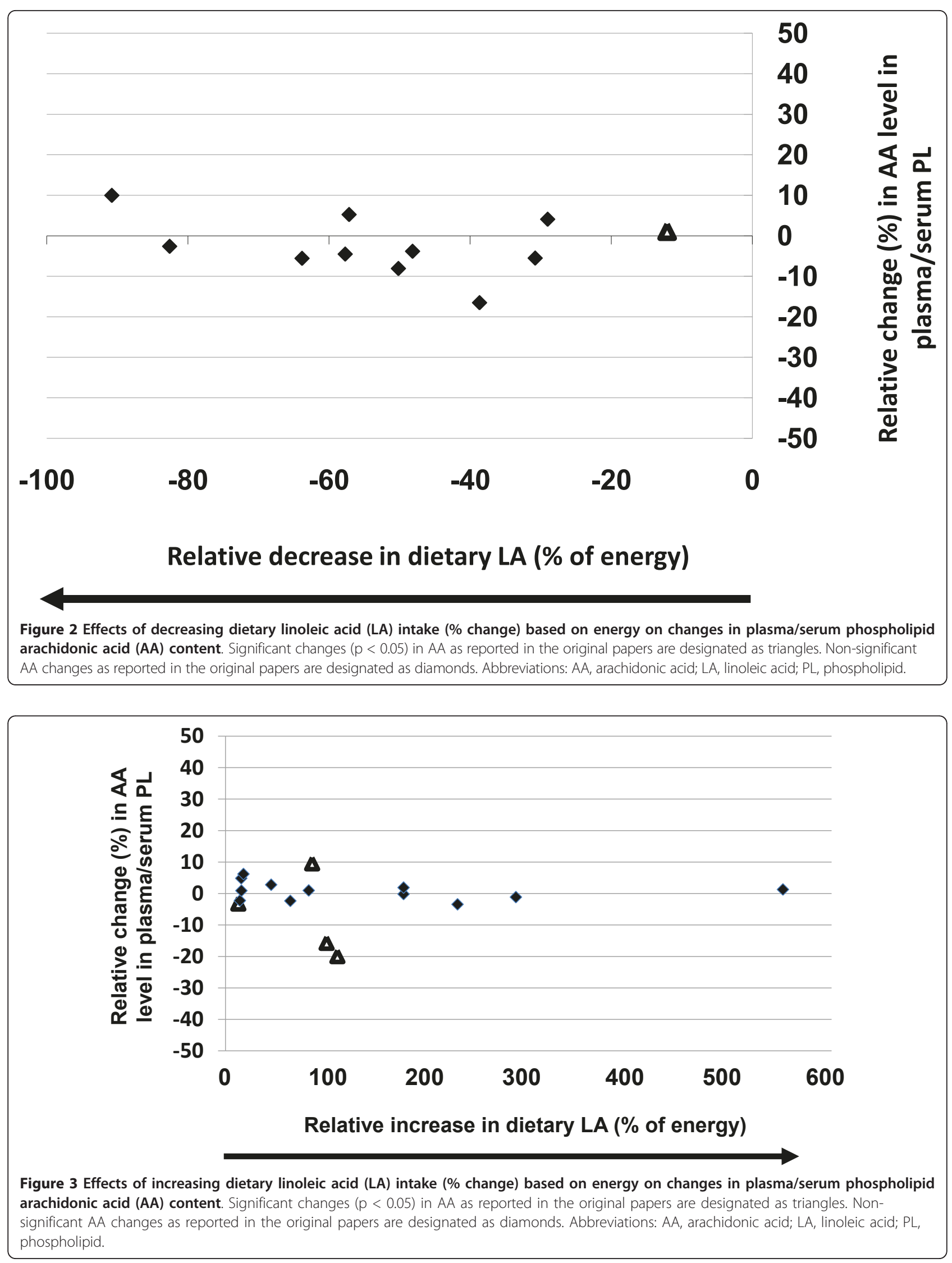




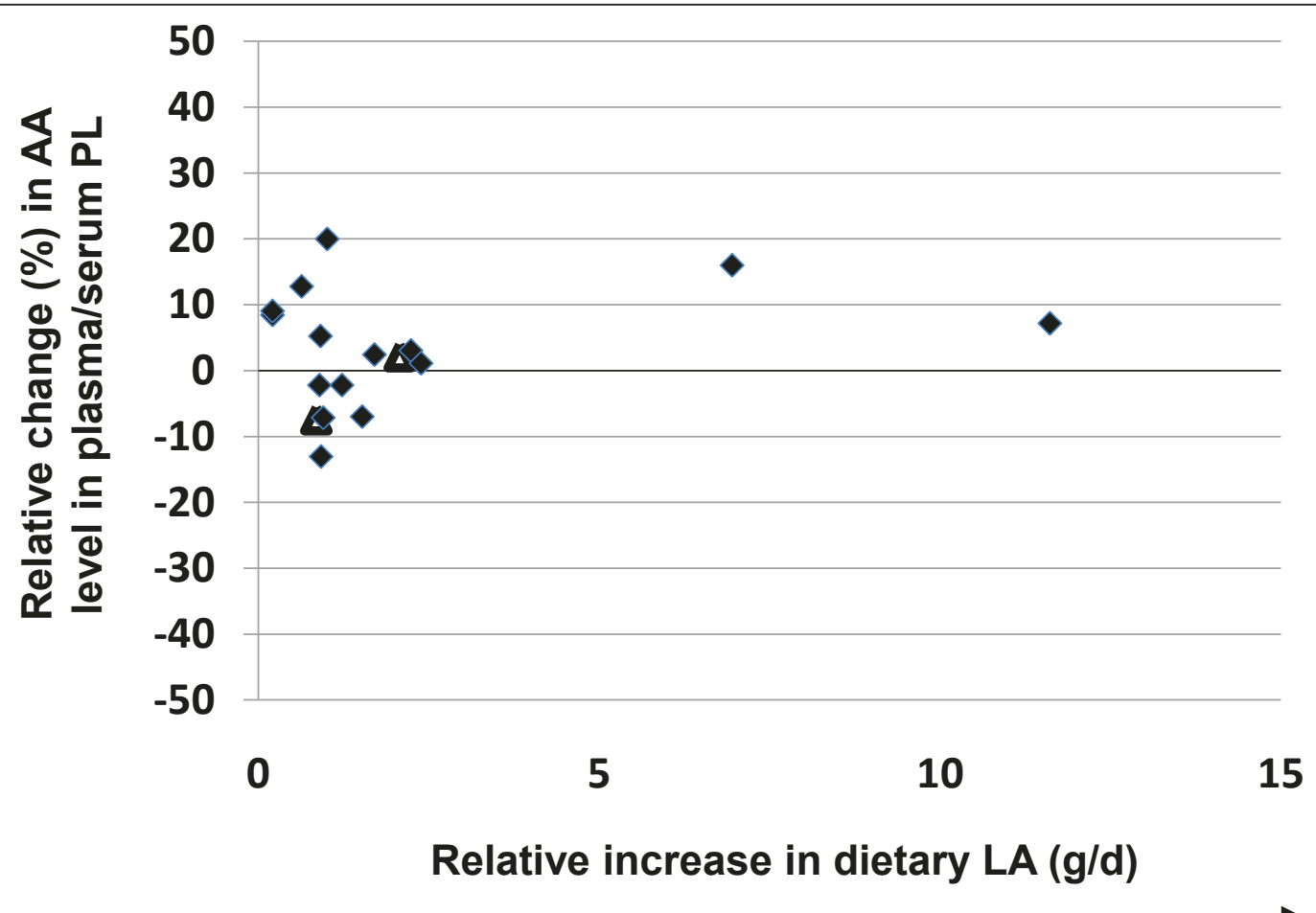

Figure 4 Effects of increasing dietary linoleic acid (LA) intake (g/d) on changes in plasma/serum phospholipid arachidonic acid (AA) content. Significant changes $(p<0.05)$ in AA as reported in the original papers are designated as triangles. Non-significant AA changes as reported in the original papers are designated as diamonds. Abbreviations: AA, arachidonic acid; LA, linoleic acid; PL, phospholipid.

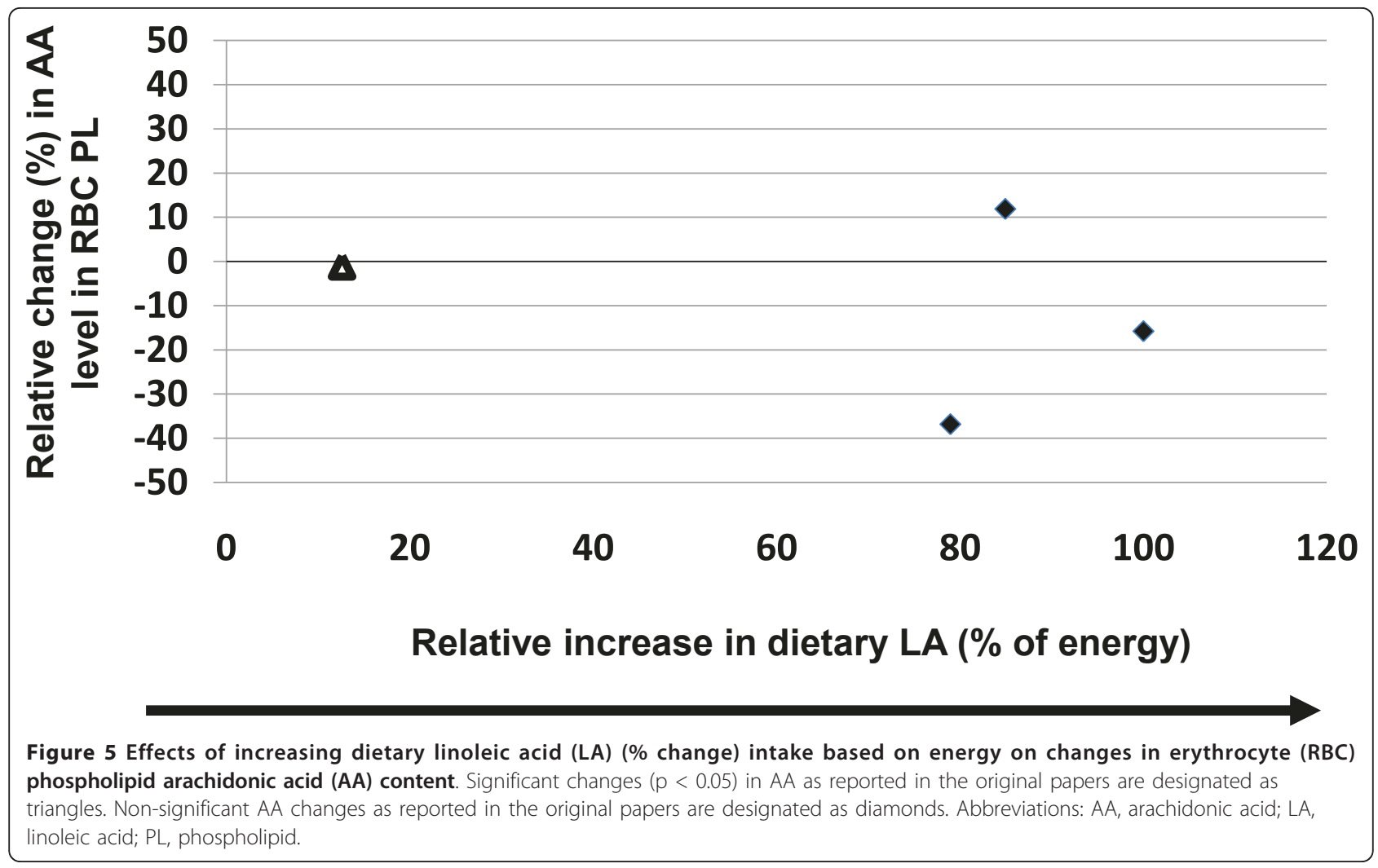




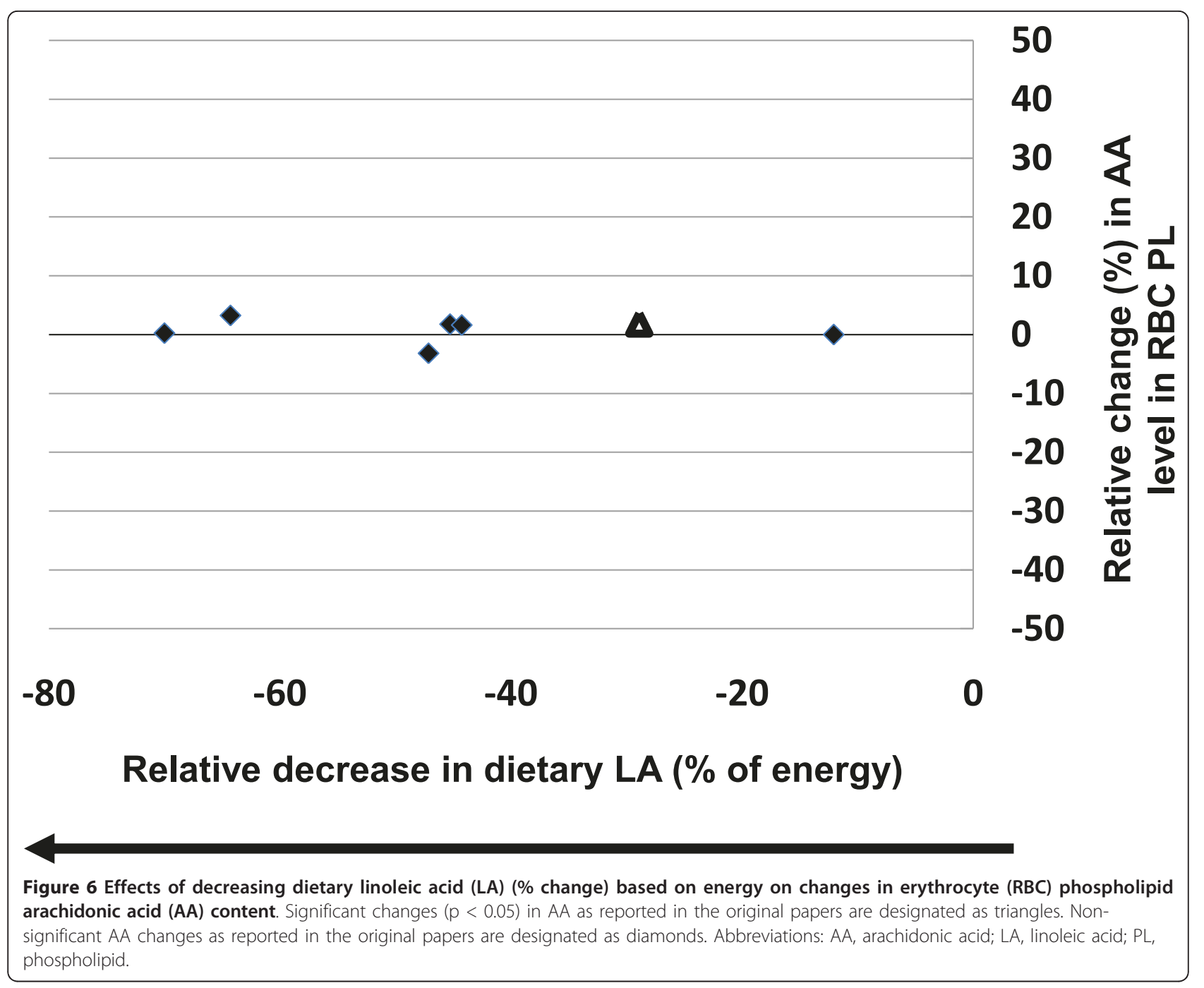

Seven studies met our criteria for the effects of GLA supplementation on changes in plasma/serum phospholipid AA content (Table 4 and Figure 7). There appeared to be a dose dependent increase in AA content with increasing intakes of GLA (ranging from $0.36 \mathrm{~g} / \mathrm{d}$ to 6.00 $\mathrm{g} /$ day). This positive correlation $\left(\mathrm{r}^{2}=0.75, \mathrm{p}=0.03, \mathrm{y}=\right.$ $0.004 x+7.36)$ was significant with a linear regression model, and approached significance with a non-linear (quadratic) regression model $\left(\mathrm{r}^{2}=0.56, \mathrm{y}=-1.4 \mathrm{x}^{2}+\right.$ $13.76 x, p=0.079)$. The number of available data may be a factor in these results. Of the six GLA data points, four of them reported statistically significant increases in AA.

Similiarly, increasing dietary AA $(0.50 \mathrm{~g} / \mathrm{d}$ to 6.00 g/day) was positively correlated with increases in plasma/serum phospholipid AA content using a quadratic regression model $\left(\mathrm{r}^{2}=0.79, \mathrm{y}=-8.7 \mathrm{x}^{2}+74.87 \mathrm{x}\right.$, $\mathrm{p}=0.013$ ) (Figure 8). All data points were reported as significantly different (Table 5).

\section{Discussion}

Arachidonic acid is arguably the most important PUFA associated with membrane phospholipids. Upon release, AA can be enzymatically metabolized to a myriad of bioactive derivatives, eicosanoids, known to contribute to a variety of chronic diseases, but are also known to be involved in tissue homeostasis and the resolution of inflammation [1-4,22]. The relative abundance of AA in membrane phospholipids positively influences eicosanoid production [23]. It is well known that dietary PUFA can affect tissue AA levels; however, what is uncertain and controversial is whether modifying current intakes of dietary LA will result in concomitant changes in tissue AA content, i.e., increasing LA intake results in an increase in tissue AA content and decreasing LA has the opposite effect [8]. The goal of this paper was to ascertain the relationship between dietary LA and tissue AA content (phospholipid pools of 


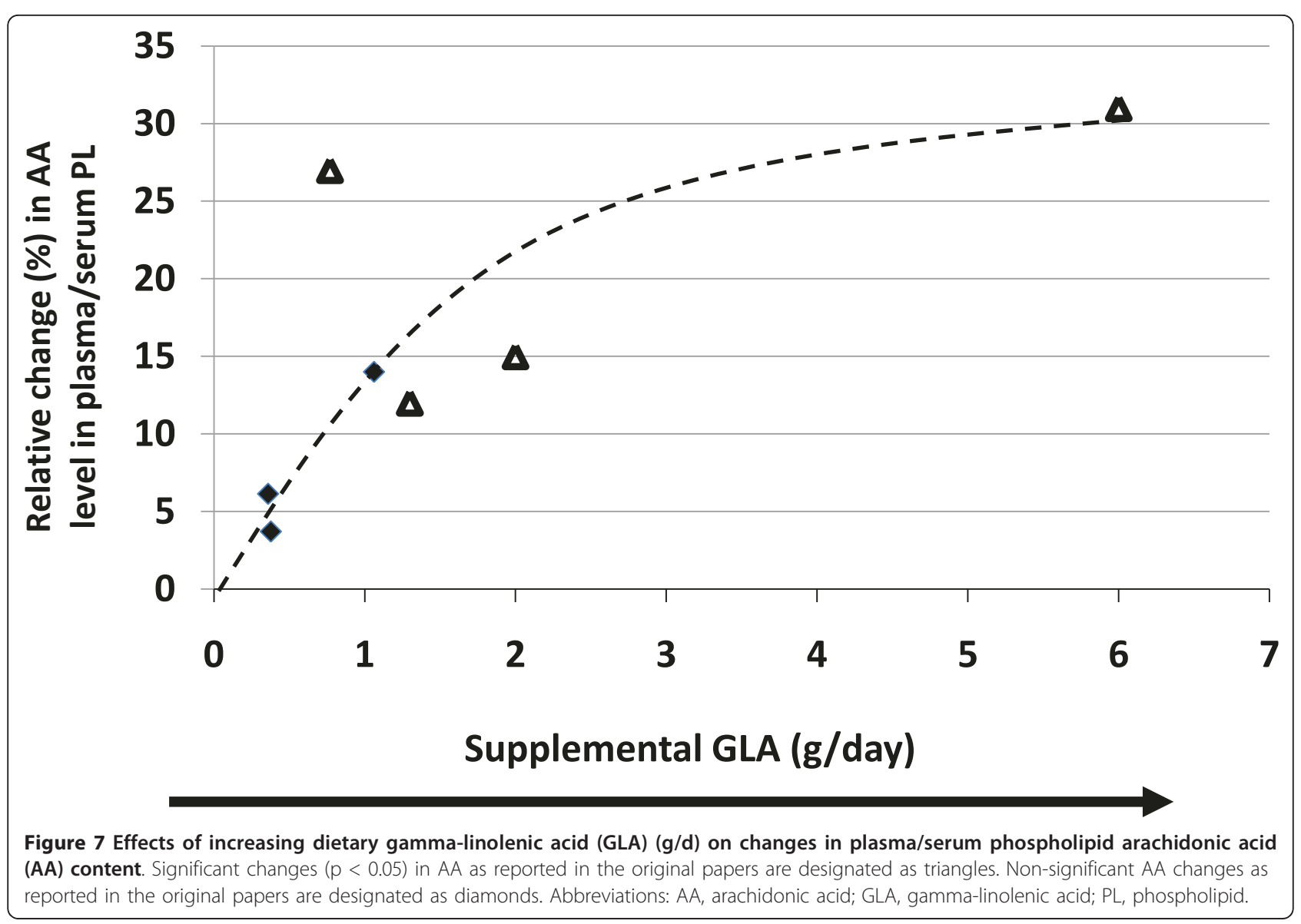

plasma/serum and erythrocytes) in adults consuming a Western-style background diet. It was not designed to address other controversies surrounding the issues of dietary n-6 or n-3 PUFA or in other population groups.

Many papers interchange the more general term n- 6 PUFA for dietary LA, but there are two major n-6 PUFA, LA and AA, that are distributed unevenly in the Western diet. While LA is the major PUFA in most commonly consumed foods, AA is exclusively found in animal products, such as, muscle, organ meats and eggs [24]. They have distinct biological activities that are biochemically linked via desatuation and elongation, and as such, LA is the conditionally essential fatty acid. Linoleic acid is specifically required in the skin to maintain the integrity of the epidermal water barrier and AA is the immediate precursor to eicosanoids, as well as being the n-6 PUFA selectively incorporated into the membranes of certain tissues, i.e., brain [25]. When consumed (LA vs. AA), they appear to have differential effects on tissue fatty acid composition, where AA appears to more robustly modify tissue AA levels and eicosanoids [14,26].

The data presented in this paper suggests that a dose response between dietary LA and tissue AA does not exists within the backdrop of individuals consuming a Western-type diet. Increasing LA by as much as $551 \%$ from baseline and reducing LA by as much as $90 \%$ from baseline failed to yield compelling evidence supporting the concept that any conversion of dietary LA to downstream metabolites results in tissue enrichment of AA, a notion commonly assumed. For example, "However, the higher concentrations of LA typically found in the Western diet results in a greater conversion of $L A$ to arachidonic acid" [8] and "Excessive n-6 precursors promotes formation of $A A$ " [9], suggesting enrichment of AA in tissues with increases in LA intake. We chose to evaluate the data by looking at changes from baseline in tissue AA content to standardize the data from one study to the next. Each study began with a baseline value and we reported percent changes from that baseline. Supplemental intakes of LA were reported based on energy and when that value could not be determined, we reported absolute supplemented values, and these data were reported seperately.

As observed from the distribution of the responses, there was wide variability. Some papers showed small increases in tissue AA levels when dietary LA changed, 


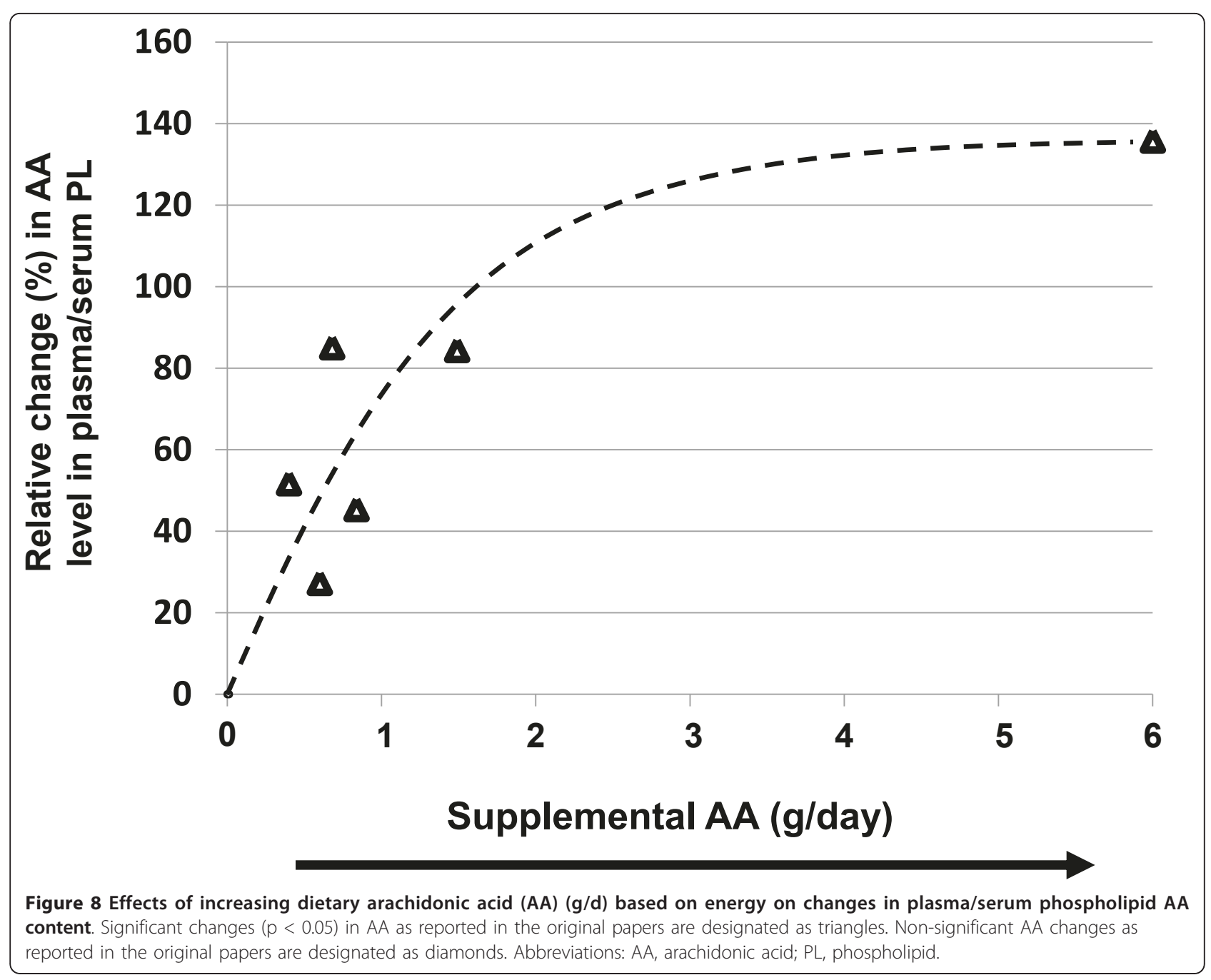

while other papers showed small decreases, but most of these changes lacked significance. When there was significance, the changes were minimal and the distribution pattern of the data did not favor an increase or a decrease. We chose plasma/serum and erythrocytes as the tissues of choice because here is where the bulk of data exists in the human literature. Erythrocytes represent a more stable pool of dietary lipids, contain very little neutral lipids and thus represents a membrane fraction of AA. Fasting plasma/serum phospholipid levels primarily (but not exclusively) represents in part phospholipids of lipoproteins that are derived from hepatic endoplasmic reticulum [15], and this pool is more responsive to more recent dietary PUFA intakes.

In an effort to identify why dietary LA may not modify tissue AA levels, we reviewed the literature for dietary GLA using the same search strategy. Was the conversion of LA to AA rate-limiting, or were tissue levels of AA saturated? Delta- 6 desaturase is the rate-limiting enzyme in the metabolism of LA to AA. GLA is a dietary n-6 PUFA that enters the metabolic pathway after the delta- 6 desaturase step. If delta- 6 desaturase is ratelimiting and tissue AA content is not saturated, then there should be evidence that including GLA in the diet increases tissue AA levels. When GLA was supplemented as the triacylglycerol form or as a component of a dietary oil containing GLA (i.e., blackcurrant, evening primrose or borage oil), tissue AA content increased in a dose responsive manner. These effects appeared to be less prominent in those studies [27-29] that used oils containing appreciable amounts of the more highly unsaturated n-3 PUFA stearidonic acid, i.e., blackcurrant [30]. When AA was supplemented in the diet, there was further enrichment in tissue AA content above that observed with either LA or GLA. These results suggest that delta- 5 desaturase potentially becomes rate limiting when GLA is supplemented. The reaction mediated by delta- 5 desaturase is an intermediate step between GLA 
and AA and by-passing that step with dietary AA leads to further enrichment. These data seem to suggest that while dietary LA maybe a metabolic precursor for AA, its influence on tissue levels in populations consuming Western diets are limited by the enzymatic conversion through delta- 6 desaturase and not due to tissue saturation of AA. These data are supported by the poor rates of conversion of plasma/serum LA to AA in adults. In tracer studies involving stable isotopes, the estimated fractional conversion of LA to AA was between $0.3 \%$ and $0.6 \%$ [31].

The levels of LA in the diet required to achieve essentiality could be as low as $0.5-2.0 \%$ of energy in infants $[32,33]$ and it has been reported that tissue levels of AA no longer respond to dietary LA intakes above $2 \%$ energy in adults [12]. Our study was designed to chose studies that incorporated a Western-type diet where LA is not typically limiting, reflective of the general public. This means a full compliment of PUFAs were being consumed along with LA supplementation. The DRIs for LA and alpha-linolenic acid (ALA, 18:3 n-3) are 12 g-17 g/d and $1.1 \mathrm{~g}-1.6 \mathrm{~g}$, respectively (women the lower figure, men the higher figure). This would be equivalent to intakes approximating $6 \%$ and $0.7 \%$ of calories per day for LA and ALA, respectively. It is not unreasonable to think that with a background diet containing LA, ALA, AA, and long-chain n-3 PUFAs, i.e. eicosapentaenoic acid (EPA, 20:5 n-3) and docosahexaenoic acid (DHA, 22:6 n-3) at typical intakes, that modifying dietary LA levels may not influence tissue AA levels. It is possible that as LA increases in the diet it maybe competing with AA for reacylation into phospholipids $[13,14,16-18,21,34]$.

A small number of studies modified LA intakes by using oils that also contained some ALA, such as soybean and canola oil $[17,35,36]$, but the results from these studies were not significant and were similar to the other results. There could be some concern that some of the supplemented oils contain ALA, such as soybean oil. It must be remembered that soybean oil has a LA:ALA ratio similar $(8: 1)$ to that found in the US diet (10:1) and if you included or excluded these papers the results were unaffected. We also included two studies that supplemented LA with recommended fish restrictions (because they met our inclusion/exclusion criteria) [19,37]. One study (+176\% LA) reported no changes in AA levels, while the other (+86\% LA) reported a $10 \%$ increase in AA.

Some of the weaknesses of this review are reflected in the studies that qualified for our evaluation. Most were not designed to specifically address our research question; however, those that were specifically designed to evaluate the effect of dietary LA on tissue AA content yielded results that were similar to the overall results [13]. Each study used a different population with potentially different background diets, but overall this would better reflect the consumption patterns of the general public. Not all studies were blinded (61\% were blinded) and dietary LA was not exclusively modified. The methods for modifying LA intakes were varied and other dietary PUFA were not controlled for with the exceptions identified previously, and data for only two tissues were evaluated. When LA was modified, it was done so by typically changing the levels of an oil rich in LA (i.e., corn oil, safflower oil, sunflower oil) or foods containing LA (as opposed to adding pure LA), reflecting how LA would be consumed by the general public. There were no standard length to the studies. For example, studies involving plasma/serum ranged between 14 days-5 months, and those looking at erythrocyte data ranged between 14-180 days. Importantly, the subjects were used as their own controls, the studies addressed changes in LA in relationship to Western-type diets, and the results were not different between those studies that were double-blind randomized placebo controlled trials (1/3) and those that were not. Despite these weaknesses, positive results were still identified with intakes of GLA and AA, helping to support those results reported with LA.

\section{Conclusions}

Elevated tissue AA levels are believed to be positively associated with eicosanoid formation and risk for a variety of chronic diseases, including cardiovascular disease, cancer and inflammation. The literature expresses concern over the fact that increasing dietary LA can potentially enrich tissues with AA due to their metabolic link. The results of this study do not support this concern. Whereas AA levels in blood phospholipids is increased by GLA or AA supplements, intervention studies bring no evidence to suggest that changes in dietary LA will modify tissue AA content in an adult population consuming a Western-type diet.

\section{Abbreviations}

AA: arachidonic acid; ALA: alpha-linolenic acid; DHA: docosahexaenoic acid; DRI: Dietary Reference Intake; EPA: eicosapentaenoic acid; GLA: gammalinolenic acid; LA: linoleic acid; NSAIDs: non-steroidal anti-inflammatory drugs; PL: phospholipid; PUFA: polyunsaturated fatty acids.

\section{Acknowledgements and funding}

This research was associated with the USDA Multistate Regional Research Project NC1039, and funded in part by the Tennessee Agricultural Experiment Station (JW).

\section{Authors' contributions}

BR conducted the research and co-wrote manuscript, and JW formulated and designed research, co-wrote manuscript and had final responsibility for all parts of the manuscript. All authors have read and approved the final manuscript. 


\section{Competing interests}

The authors declare that they have no competing interests.

Received: 4 March 2011 Accepted: 10 June 2011

Published: 10 June 2011

\section{References}

1. Calder PC: n-3 Polyunsaturated fatty acids, inflammation, and inflammatory diseases. Am J Clin Nutr 2006, 83:S1505-1519.

2. Wang D, DuBois RN: Prostaglandins and cancer. Gut 2006, 55:115-122.

3. McEntee MF, Whelan J: Dietary polyunsaturated fatty acids and colorectal neoplasia. Biomed Pharmacother 2002, 56:380-387.

4. Allayee $\mathrm{H}$, Roth $\mathrm{N}$, Hodis HN: Polyunsaturated fatty acids and cardiovascular disease: implications for nutrigenetics. J Nutrigenet and Nutrigenomics 2009, 2:140-148.

5. Institute of Medicine, Food and Nutrition Board. Dietary reference Intakes for energy, carbohydrate, fiber, fat, fatty acids, cholesterol, protein, and amino acids. [http://fnic.nal.usda.gov/nal_display/index.php? info_center=4\&tax_level=3\&tax_subject=274\&topic_id=1323\&level3_

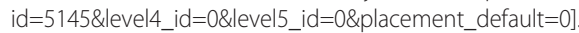

6. Mohrhauer H, Holman RT: The effect of dose level of essential fatty acids upon fatty acid composition of the rat liver. J Lipid Res 1963, 4:151-159.

7. Renaud SC, Ruf JC, Petithory D: The positional distribution of fatty acids in palm oil and lard influences their biologic effects in rats. J Nutr 1995, 125:229-237.

8. Wall RR, Paul R, Fitzgerald, Gerald F, Stanton, Catherine : Fatty acids from fish: the anti-inflammatory potential of long-chain omega-3 fatty acids. Nutr Rev 2010, 68:280-289.

9. Layé S: Polyunsaturated fatty acids, neuroinflammation and well being. Prostaglandins Leukot and Essent Fatty Acids 2010, 82:295-303.

10. Simopoulos AP: The importance of the omega-6/omega-3 fatty acid ratio in cardiovascular disease and other chronic diseases. Exp Biol Med 2008, 233:674-688.

11. Lands WE: Dietary fat and health: the evidence and the politics of prevention: careful use of dietary fats can improve life and prevent disease. Ann N Y Acad Sci 2005, 1055:179-192.

12. James M, Gibson R, D'Angelo M, Neumann M, Cleland L: Simple relationships exist between dietary linoleate and the $n-6$ fatty acids of human neutrophils and plasma. Am J Clin Nutr 1993, 58:497-500.

13. Liou YA, Innis SM: Dietary linoleic acid has no effect on arachidonic acid, but increases $n-6$ eicosadienoic acid, and lowers dihomo-[gamma]linolenic and eicosapentaenoic acid in plasma of adult men. Prostaglandins Leukot and Essent Fatty Acids 2009, 80:201-206.

14. O Adam AT, Zollner N: Influence of dietary linoleic acid intake with different fat intakes on arachidonic acid concentrations in plasma and platelet lipids and eicosanoid biosynthesis in female volunteers. Ann Nutr Metab 2003, 47:31-36.

15. Shelness GS, Sellers JA: Very-low-density lipoprotein assembly and secretion. Current Opinion in Lipidology 2001, 12:151-157.

16. King IB, Lemaitre RN, Kestin M: Effect of a low-fat diet on fatty acid composition in red cells, plasma phospholipids, and cholesterol esters: investigation of a biomarker of total fat intake. Am J Clin Nutr 2006, 83:227-236.

17. Lasserre M, Mendy F, Spielmann D, Jacotot B: Effects of different dietary

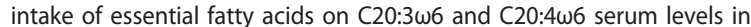
human adults. Lipids 1985, 20:227-233.

18. Raatz SK BD, Thomas W, Kris-Etherton P: Total fat intake modifies plasma fatty acid composition in humans. J Nutr 2001, 131:231-234.

19. Valsta LM SI, Aro A, Mutanen M: Alpha-linolenic acid in rapeseed oil partly compensates for the effect of fish restriction on plasma long chain $n-3$ fatty acids. Eur J Clin Nutr 1996, 50:229-235.

20. Grimsgaard S, Bonaa K, Hansen J, Nordoy A: Highly purified eicosapentaenoic acid and docosahexaenoic acid in humans have similar triacylglycerol-lowering effects but divergent effects on serum fatty acids. Am J Clin Nutr 1997, 66:649-659.

21. Geppert J, Demmelmair H, Hornstra G, Koletzko B: Co-supplementation of healthy women with fish oil and evening primrose oil increases plasma docosahexaenoic acid, $\gamma$-linolenic acid and dihomo- $\gamma$-linolenic acid levels without reducing arachidonic acid concentrations. Br J Nutr 2008, 99:360-369.
22. Ryan A, Godson C: Lipoxins: regulators of resolution. Current Opinion in Pharmacology 2010, 10:166-172.

23. Whelan J, McEntee MF: Dietary ( $\mathrm{n}-6)$ PUFA and intestinal tumorigenesis. $J$ Nutr 2004, 134:3421S-3426.

24. Taber $\mathrm{L}$, Chiu $\mathrm{CH}$, Whelan J: Assessment of the arachidonic acid content in foods commonly consumed in the American diet. Lipids 1998, 33:1151-1157.

25. Rapoport SI: Brain arachidonic and docosahexaenoic acid cascades are selectively altered by drugs, diet and disease. Prostaglandins Leukot and Essent Fatty Acids 2008, 79:153-156.

26. Whelan J, Surette ME, Hardardottir I, Lu G, Golemboski KA, Larsen E, Kinsella JE: Dietary arachidonate enhances tissue arachidonate levels and eicosanoid production in syrian hamsters. J Nutr 1993, 123:2174-2185.

27. Miles EA, Banerjee T, Calder PC: The influence of different combinations of [gamma]-linolenic, stearidonic and eicosapentaenoic acids on the fatty acid composition of blood lipids and mononuclear cells in human volunteers. Prostaglandins Leukot and Essent Fatty Acids 2004, 70:529-538.

28. Mills De PK, Harvey KA, Ward RP: Dietary fatty acid supplementation alters stress reactivity and performance in man. $J$ Hum Hypertens 1989, 3:111-116.

29. Tahvonen RL, Schwab US, Linderborg KM, Mykkänen HM, Kallio HP: Black currant seed oil and fish oil supplements differ in their effects on fatty acid profiles of plasma lipids, and concentrations of serum total and lipoprotein lipids, plasma glucose and insulin. J Nutr Biochem 2005, 16:353-359.

30. Whelan J: Dietary Stearidonic Acid Is a Long Chain (n-3) Polyunsaturated Fatty Acid with Potential Health Benefits. The Journal of Nutrition 2009, 139:5-10.

31. Demmelmair, Iser, Rauh P, Koletzko : Comparison of bolus versus fractionated oral applications of $[13 \mathrm{C}]$-linoleic acid in humans. Eur J Clin Invest 1999, 29:603-609.

32. Paulsrud JR, Pensler L, Whitten CF, Stewart S, Holman RT: Essential fatty acid deficiency in infants induced by fat-free intravenous feeding. Am J Clin Nutr 1972, 25:897-904.

33. Cuthbertson W: Essential fatty acid requirements in infancy. Am J Clin Nutr 1976, 29:559-568.

34. Yaqoob PPH, Cortina-Borja M, Newsholme EA, Calder PC: Encapsulated fish oil enriched in alpha-tocopherol alters plasma phospholipid and mononuclear cell fatty acid compositions but not mononuclear cell functions. Eur J Clin Invest 2000, 30:260-274.

35. Lichtenstein AH, Matthan NR, Jalbert SM, Resteghini NA, Schaefer EJ Ausman LM: Novel soybean oils with different fatty acid profiles alter cardiovascular disease risk factors in moderately hyperlipidemic subjects. Am J Clin Nutr 2006, 84:497-504.

36. Vega-Lopez S, Ausman LM, Jalbert SM, Erkkila AT, Lichtenstein AH: Palm and partially hydrogenated soybean oils adversely alter lipoprotein profiles compared with soybean and canola oils in moderately hyperlipidemic subjects. Am J Clin Nutr 2006, 84:54-62.

37. Angela Liou Y, King DJ, Zibrik D, Innis SM: Decreasing linoleic acid with constant \{alpha\}-linolenic acid in dietary fats increases $(n-3)$ eicosapentaenoic acid in plasma phospholipids in healthy men. J Nutr 2007, 137:945-952

38. Goyens PL, Spilker ME, Zock PL, Katan MB, Mensink RP: Conversion of \{alpha\}-linolenic acid in humans is influenced by the absolute amounts of \{alpha\}-linolenic acid and linoleic acid in the diet and not by their ratio. Am J Clin Nutr 2006, 84:44-53.

39. Li D, Sinclair A, Wilson A, Nakkote S, Kelly F, Abedin L, Mann N, Turner A: Effect of dietary \{alpha\}-linolenic acid on thrombotic risk factors in vegetarian men. Am J Clin Nutr 1999, 69:872-882.

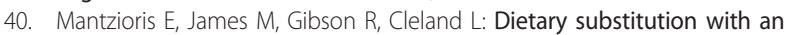
alpha-linolenic acid-rich vegetable oil increases eicosapentaenoic acid concentrations in tissues. Am J Clin Nutr 1994, 59:1304-1309.

41. Geppert J, Kraft V, Demmelmair H, Koletzko B: Docosahexaenoic acid supplementation in vegetarians effectively increases omega-3 index: A randomized trial. Lipids 2005, 40:807-814.

42. Thijssen MAMA, Hornstra G, Mensink RP: Stearic, oleic, and linoleic acids have comparable effects on markers of thrombotic tendency in healthy human subjects. J Nutr 2005, 135:2805-2811.

43. Montoya MT, Porres A, Serrano S, Fruchart JC, Mata P, Gerique JAG, Castro GR: Fatty acid saturation of the diet and plasma lipid 
concentrations, lipoprotein particle concentrations, and cholesterol efflux capacity. Am J Clin Nutr 2002, 75:484-491.

44. Andersson A, Nalsen C, Tengblad S, Vessby B: Fatty acid composition of skeletal muscle reflects dietary fat composition in humans. Am J Clin Nutr 2002, 76:1222-1229.

45. Thies F, Nebe-von-Caron G, Powell JR, Yaqoob P, Newsholme EA, Calder PC: Dietary supplementation with eicosapentaenoic acid, but not with other long-chain $n-3$ or $n-6$ polyunsaturated fatty acids, decreases natural killer cell activity in healthy subjects aged $>55$ y. Am J Clin Nutr 2001, 73:539-548.

46. Johansson AK, Korte H, Yang B, Stanley JC, Kallio HP: Sea buckthorn berry oil inhibits platelet aggregation. J Nutr Biochem 2000, 11:491-495.

47. Kew S, Mesa MD, Tricon S, Buckley R, Minihane AM, Yaqoob P: Effects of oils rich in eicosapentaenoic and docosahexaenoic acids on immune cell composition and function in healthy humans. Am J Clin Nutr 2004, 79:674-681.

48. Buckley R, Shewring B, Turner R, Yaqoob P, Minihane AM: Circulating triacylglycerol and apoE levels in response to EPA and docosahexaenoic acid supplementation in adult human subjects. Br J Nutr 2004, 92:477-483.

49. Wallace FA, Miles EA, Calder PC: Comparison of the effects of linseed oil and different doses of fish oil on mononuclear cell function in healthy human subjects. Br J Nutr 2003, 89:679-689.

50. Conquer JA, Holub BJ: Supplementation with an algae source of docosahexaenoic acid increases ( $\mathrm{n}-3)$ fatty acid status and alters selected risk factors for heart disease in vegetarian subjects. J Nutr 1996, 126:3032-3039.

51. Finnegan YE, Minihane AM, Leigh-Firbank EC, Kew S, Meijer GW, Muggli R, Calder PC, Williams CM: Plant- and marine-derived $n-3$ polyunsaturated fatty acids have differential effects on fasting and postprandial blood lipid concentrations and on the susceptibility of LDL to oxidative modification in moderately hyperlipidemic subjects. Am J Clin Nutr 2003, 77:783-795.

52. Ebden P, Bevan C, Banks J, Fennerty A, Walters EH: A study of evening primrose seed oil in atopic asthma. Prostaglandins, Leukot and Essent Fatty Acids 1989, 35:69-72.

53. Johnson MM, Swan DD, Surette ME, Stegner J, Chilton T, Fonteh AN, Chilton FH: Dietary Supplementation with gamma -linolenic acid alters fatty acid content and eicosanoid production in healthy humans. J Nutr 1997, 127:1435-1444.

54. Sinclair AJ, Mann NJ: Short-term diets rich in arachidonic acid influence plasma phospholipid polyunsaturated fatty acid levels and prostacyclin and thromboxane production in humans. J Nutr 1996, 126:1110S-1114.

55. Ishikura Y, Ikeda G, Akimoto K, Hata M, Kusumoto A, Kidokoro A, Kontani M, Kawashima H, Kiso Y, Koga Y: Arachidonic acid supplementation decreases P300 latency and increases P300 amplitude of event-related potentials in healthy elderly men. Neuropsychobiology 2009, 60:73-79.

56. Kusumoto A, Ishikura Y, Kawashima H, Kiso Y, Takai S, Miyazaki M: Effects of arachidonate-enriched triacylglycerol supplementation on serum fatty acids and platelet aggregation in healthy male subjects with a fish diet. Br J Nutr 2007, 98:626-635.

57. Nelson G, Schmidt P, Bartolini G, Kelley D, Phinney S, Kyle D, Silbermann S, Schaefer $E$ : The effect of dietary arachidonic acid on plasma lipoprotein distributions, apoproteins, blood lipid levels, and tissue fatty acid composition in humans. Lipids 1997, 32:427-433.

58. Seyberth HW OO, Kennedy T, Sweetman BJ, Danon A, Frölich JC, Heimberg M, Oates JA: Increased arachidonate in lipids after administration to man: effects on prostaglandin biosynthesis. Clin Pharmacol Ther 1975, 18:521-529.

doi:10.1186/1743-7075-8-36

Cite this article as: Rett and Whelan: Increasing dietary linoleic acid does not increase tissue arachidonic acid content in adults consuming Western-type diets: a systematic review. Nutrition \& Metabolism 2011 8:36.

\section{Submit your next manuscript to BioMed Central and take full advantage of:}

- Convenient online submission

- Thorough peer review

- No space constraints or color figure charges

- Immediate publication on acceptance

- Inclusion in PubMed, CAS, Scopus and Google Scholar

- Research which is freely available for redistribution

Submit your manuscript at www.biomedcentral.com/submit 